\title{
¿POURQUOI TU TE METS LÀ COMME ÇA? MIGRANTES CONGOLEÑAS Y PRÁCTICAS DE HACIENDO- LUGAR EN SÃO PAULO
}

\author{
Pourquoi tu te mets là comme ça? Congolese migrants \\ and everyday place-making in São Paulo
}

Franziska Reiffen*

\begin{abstract}
Resumen. En este artículo abordo el tema del haciendo-lugar en situaciones de desplazamiento desde la perspectiva de migrantes provenientes de la República Democrática del Congo quienes viven en la ciudad de São Paulo. A través de un abordaje etnográfico, analizo las experiencias de mujeres migrantes en un lugar de trabajo y en una parroquia evangélica. Mi intención es hacer ver cómo estas migrantes (trans)forman lugares en la ciudad, cómo se insertan en redes existentes y a qué dificultades se enfrentan en su entorno marcado por relaciones desiguales de poder.
\end{abstract}

Palabras clave: haciendo-lugar; desplazamiento; São Paulo; migración congoleña.

Abstract. In this article, I focus on place-making in situations of displacement from the perspective of migrants from the Democratic Republic of Congo who live in São Paulo. Drawing on ethnographic research, I analyze the experiences of female migrants in a working place and in an evangelic church. I aim to show how migrants (trans)form places in the city, how they become part of existing networks and what difficulties they face in a surrounding shaped by unequal power relations.

Keywords: place-making; displacement; São Paulo; Congolese migration.

Instituto de Antropología y de Estudios Africanos de la Universidad Johannes Gutenberg de Mainz, Alemania. Mainz, Alemania. E-mail: reiffen@uni-mainz.de. Orcid: 0000-0001-91184308. 


\section{Introducción}

El 29 de marzo de 2017, un conocido me presentó Thérèse ${ }^{1}$, una migrante congoleña quien trabajaba en una peluquería en el "Centro Comercial Presidente", también Ilamado "Galeria Presidente", en el centro de São Paulo. Thérèse estaba atendiendo una cliente, y mientras yo esperaba que tenga tiempo para conversar conmigo, me puse a charlar con otra señora sentada en la peluquería. Se presentó como Gabrièle y era la hermana mayor de Thérèse. Gabrièle había llegado hace dos semanas a São Paulo desde Angola, donde había pasado los últimos años de su vida. Estaba sentada en la peluquería porque quería trabajar como peluquera, como su hermana menor. El problema era que Gabrièle no conocía las técnicas necesarias para hacer peinados. Pasaba sus días sentada en la peluquería, observando, aprendiendo y aburriéndose. Para mí, una antropóloga en busca de migrantes dispuestos/-as a dejarme participar en sus actividades y con tiempo para conversar conmigo, el encuentro con Gabrièle era un golpe de suerte. Gabrièle tenía tiempo, un tiempo que parecía pasar un poco más rápido si ella podía ocuparse de mí. Durante dos semanas compartió conmigo sus impresiones de lo que estaba pasando en su entorno y trató de explicarme cómo funcionaba la vida de la peluquería según ella lo entendía. Incluso se volvió casi una asistente de investigación, sacando fotos con mi celular y traduciendo conversaciones del lingala al francés o al portugués.

¿Cómo encontrar y hacer su lugar en una ciudad como São Paulo? Cuando nos conocimos, Gabrièle estaba empezando a descubrir la ciudad, a insertarse en ciertas redes a las cuales tenía acceso gracias a su hermana, a crear sus propias redes y a definir su lugar en las configuraciones sociales complejas que constituían su nuevo entorno. Este proceso del "haciendolugar" (place-making, Escobar, 2010) es el foco del análisis de este artículo, así como de este dossier temático. Según autores como Escobar (2000) y Lems (2018), el lugar y las prácticas que contribuyen a un haciendo-lugar siguen siendo relevantes a pesar del enfoque reciente de las ciencias sociales en la movilidad y en el desplazamiento. Obviamente, esta relevancia no se limita a personas recién llegadas, ni a personas migrantes. La necesidad del haciendolugar es, más bien, una necesidad existencial de los seres humanos (Lems, 2018). Al mismo tiempo, cada persona puede experimentar la pérdida de lugar y la sensación de estar desplazada. Me baso entonces en una concepción amplia de desplazamiento, considerándolo, como sugieren Drotbohm y Lems, "una condición empujada por historias de guerras, colonialismo y conflictos violentos y [...] una metáfora conceptual para un sentido existencial de ruptura

\footnotetext{
A fin de mantener el anonimato de mis interlocutoras - quienes al momento de realizar la investigación, se encontraban mayoritariamente en proceso de solicitud de refugio - todos sus nombres han sido cambiados.
} 
y alienación" (Drotbohm, Lems, 2018, traducción de la autora, resaltados del original). De ahí, ser una persona desplazada no significa necesariamente estar en movimiento, un desplazamiento se puede igualmente producir por discursos y prácticas que hacen que una persona se sienta "fuera de lugar" sin moverse realmente (Siu, 2007).

Sin embargo, en este artículo sí me enfocaré en las prácticas de personas migrantes. Sobre todo, la perspectiva propuesta aquí acentúa la relación entre procesos de desplazamiento y la necesidad de hacerse lugares sociales. Se explora cómo personas en una situación de desplazamiento se esfuerzan por establecerse, asentarse y crear redes sociales dentro de las restricciones y las oportunidades específicas de la localidad en la cual se encuentran (Çağlar, Glick Schiller, 2018). Al mismo tiempo, las personas migrantes empiezan también a (trans)formar los lugares donde se encuentran (Pelican, 2014). De acuerdo con Bjarnesen y Vigh (2016), el haciendo-lugar no significa una simple "localización física", sino que incluye todas las prácticas con las cuales las personas "bregan por estar situadas de manera positiva en un paisaje relacional" (Bjarnesen, Vigh, 2016, p. 10, traducción de la autora). En relación a la migración, esta perspectiva se enfoca más en la "situación" de las personas migrantes que en su movilidad, es decir, en las maneras en las que éstas forman y transforman los lugares en los que se encuentran y en las dimensiones afectivas y materiales de su inserción en ellos (Lems, 2018; Drotbohm, Winters, 2018; Winters, Reiffen, en este número).

El presente artículo aborda principalmente las prácticas y perspectivas de Gabrièle en tanto recién llegada a São Paulo, que son complementadas con las perspectivas de otras personas migrantes cuya estadía en esta ciudad fue más larga. La mayoría de mis interlocutores/-as eran de la República Democrática del Congo (RDC) y algunos/-as habían migrado a otros países subsaharianos antes de llegar a Brasil. São Paulo es una de las ciudades en América del Sur que se convirtieron en un núcleo importante para muchas personas africanas que migran, ya sea como lugar definitivo o de paso hacia otro. En esta ciudad la migración africana contemporánea sin duda ha dejado sus huellas. La Galeria Presidente donde conocí a Gabrièle se destaca como un lugar que ofrece posibilidades para trabajar y sociabilizar a muchos/-as migrantes africanos/-as. Para las personas que trabajan y pasan su tiempo aquí, este centro comercial está vinculado con otros lugares cercanos y lejanos. Al mismo tiempo, está atravesado y configurado por redes sociales complejas que uno tiene que conocer y comprender para poder adentrarse en ellas. También está enmarcado por un contexto de trabajo informal y muchas veces precario. A continuación, analizaré desde una perspectiva del haciendo-lugar cómo las personas migrantes intentan formar parte de este contexto social- y económicamente complejo, cómo forman y transforman su entorno material y 
social, cómo se relacionan, así como sus relaciones de poder y las dificultades que puede tener una recién llegada en este entorno.

El análisis se basa en datos recogidos durante una investigación exploratoria en marzo y abril 2017 en la ciudad de São Paulo. Esta investigación es parte de un proyecto de doctorado que examina procesos del relacionarse en múltiples situaciones de desplazamiento (Reiffen, 2018). Mientras que la mayor parte de la investigación doctoral fue realizada en Argentina en los años 2017 y 2018, la investigación exploratoria en São Paulo me permitió conocer y comparar otro contexto de llegada para migrantes provenientes de distintos países africanos. Durante tres semanas llevé a cabo observaciones participantes en lugares frecuentados por migrantes, mayoritariamente de origen congoleño, en Brás, en el centro antiguo de São Paulo y en la llamada "Zona Este" de la ciudad, incluyendo bares, iglesias y el centro comercial en el cual trabajan las personas mencionadas en este artículo. Realicé también conversaciones informales con aquellas personas migrantes y con personas trabajando en centros de acogida de migrantes, permitiéndome ganar una perspectiva más amplia de la situación de las personas migrantes en la ciudad.

\section{Migración africana contemporánea a São Paulo}

Gabrièle y su hermana Thérèse son dos de numerosas personas migrantes africanas que han llegado desde el fin del siglo XX a Brasil. Después de haber acogido un número significativo de migrantes africanos en la época de los conflictos de independencia y post-independencia, sobre todo de otros países lusófonos como Angola (Patarra, Fernandes, 2011), Brasil se ha vuelto actualmente uno de los países receptores principales de la migración africana hacia América del Sur (OIM, 2012).

La mayoría de las personas migrantes procediendo del África subsahariano que llegaron desde el fin del siglo XX a Brasil es de nacionalidad angoleña, seguida por las de la RDC (Vieira, 2015). Más recientemente, en particular desde el 2012, creció la migración desde Senegal a Brasil (Redin, Bittencourt Minchola, 2015). Además, Brasil ha recibido un número de refugiados elevado en comparación con otros países de la región. En el 2017, según datos del Ministerio de Justicia, las personas que solicitaron refugio en Brasil venían sobre todo de otros países latinoamericanos, como Venezuela, Cuba o Haití, al igual que de otros países africanos, principalmente Angola, seguido por Senegal, Nigeria, y la RDC. Las mismas estadísticas muestran que de los 587 refugiados reconocidos por el Comitê Nacional para os Refugiados (CONARE) en 2017, 106 fueron de la RDC (Ministério da Justiça, 2018). En São Paulo, el Centro de Referência para Refugiados de la Caritas atendió en 2017 a un total de 6.397 personas, y destacó que los países de origen son sobre todo Angola y Síria, y, en tercer lugar, la RDC (Carvalho, 2018). Considerando la 
relativa facilidad de obtener una "visa de visita" en Brasil, muchas personas suelen entrar al país como turistas y después realizan los trámites para solicitar refugio (Vieira, 2015) que duran en general entre uno y dos años. Con la solicitud de refugio hecho, las personas migrantes pueden obtener, entre otros, un permiso de trabajo (Redin, Bittencourt Minchola, 2015; Silva et alii, 2018). Según un análisis de las estadísticas de la Policía Federal de los años 2010 a 2015, realizado por Silva y colegas, la mayoría de las personas provenientes de la RDC durante este tiempo solicitó refugio y un 55\% de ellas fueron reconocidas como refugiados. Otras solicitaron visados de estudiantes o tramitaron la reunificación familiar (Silva et alii, 2018, p. 448).

Los proyectos migratorios y la motivación inicial para emprender el viaje transatlántico varían según los contextos de origen y los individuos. En el caso de la migración desde la RDC, una de las razones principales es el conflicto armado continuo en el país, así como las condiciones económicas desfavorables. Muchas personas migrantes de la RDC, así como de otros países africanos pasan por o viven en otros países antes de llegar en Brasil (Baeninger, Guimarães Peres, 2011). Eso fue también el caso de varias migrantes de la RDC que encontré en Brasil: Ellas habían vivido en países vecinos, como Angola, antes de optar por la migración transcontinental. Incluso, algunas habían obtenido otros títulos de residencia o hasta la nacionalidad en países terceros. Cuando las contacté por primera vez, se encontraban mayoritariamente en un proceso de solicitar refugio. La solicitud de refugio consiste en que las personas solicitantes presentan ante las autoridades brasileñas un relato individual que demuestra de forma convincente por qué esta persona puede contar como haber emprendido una migración "forzada" (Rossa, Menezes, 2018) ${ }^{2}$. En nuestras interacciones, mis preguntas sobre las motivaciones y trayectos de migración causaron conflictos entre mis interlocutoras porque algunas no querían compartir informaciones que podrían amenazar su elegibilidad al estatus de refugiado. Por eso, decidí no elaborar sobre las motivaciones y trayectos individuales. En general, es importante subrayar que no existe siempre una única motivación para la migración, y eso no solamente vale para personas migrantes africanas en Brasil, sino que para cualquiera que decida tomar la decisión. Más bien, en las trayectorias de muchas personas

2 Como señalan Rossa y Menezes (2018), las solicitudes de refugio se tratan a veces de manera distinta por las autoridades según la nacionalidad de los/las solicitantes. Siguiendo al terremoto (2010) y a un huracán (2012) que devastaron a Haití, Brasil creó un visado humanitario, posibilitando a muchos/-as haitianos/-as obtener el estatus de refugio ambiental en Brasil. También, las solicitudes de sírios/-as fueron, por un tiempo, reconocidas inmediatamente. En cambio, las solicitudes de congoleños/-as están analizadas caso por caso y muchas veces deferidas. Las autoras subrayan que estas "diferenças de tratamento no reconhecimento de status e ao conferir proteção não se explicam por fundamentos jurídicos, mas sim, por critérios políticos, dos quais a criação e identificação em categorias pode agregar proteção ou não" (Rossa, Menezes, 2018, p. 393). 
migrantes, los efectos de conflictos políticos armados, necesidades económicas y otras consideraciones personales, sociales y culturales se suman; en ciertos momentos siendo uno u otro la mayor motivación para quedarse o para seguir el trayecto. Entonces, una distinción clara entre "migrantes" y "refugiados", o entre migración "voluntaria" y "forzada", como la exigen actores políticos y las leyes de migración, no es posible y tampoco debería adaptarse, sin indagar su significado y uso, por las ciencias sociales (Rossa, Menezes, 2018).

En el contexto de la reciente migración africana, grandes ciudades como São Paulo y Buenos Aires se han vuelto puntos importantes en las rutas migratorias que atraviesan América del Sur (OIM, 2010). São Paulo es una ciudad que ha atraído y sido transformada por múltiples migraciones, y que posee de estructuras de acogida y de orientación para migrantes recién llegados/-as, así como de movimientos sociales organizados luchando para los derechos de migrantes (Sato, 2017). En la ciudad, las personas migrantes africanas han empezado a transformar ciertas localidades, han establecido sus redes y han formado sus propias organizaciones.

\section{Haciendo-lugar a través del comercio y la religión}

Referirnos al haciendo-lugar de personas migrantes no significa retomar un discurso esencializante sobre el arraigo, como subrayamos en el artículo introductorio a este dossier temático (Winters y Reiffen, en este número), sino reconocer una realidad empírica: acordamos con Pardue (2017) al reconocer que las personas migrantes transforman las ciudades contemporáneas y dejan allí sus huellas, no a contramano de su movilidad o sus enlaces transnacionales, sino como parte integral de la globalización actual. El mismo autor distingue diversos tipos de lugares en los cuales personas migrantes africanas transforman actualmente la ciudad de São Paulo, entre ellos sitios comerciales y religiosos (Pardue, 2017).

El lugar destacado que adquieren los mercados o centros comerciales en relación con las migraciones se encuentra en la literatura sobre la reciente migración africana en São Paulo, al igual que en relación con otras ciudades y otras migraciones. Por ejemplo, se pone de relieve el rol de personas migrantes en el funcionamiento de "mega-ferias" famosas como la "Feira da Madrugada" en São Paulo (Amrith, 2018), "La Salada" en Buenos Aires (Gago, 2014) o "Chungking Mansions" en Hong Kong (Mathews, 2011). Estas ferias están en el centro de un comercio global y son el resultado de una globalización a veces llamada "desde abajo" (Mathews et alii, 2012) 3 .

\footnotetext{
3 La perspectiva de la "globalización desde abajo" ha sido criticada por solidificar imaginaciones del comercio en el "sur global" como informal o directamente ilegal (Röschenthaler, Jedlowski, 2017, p. 6-7).
} 
No obstante, el presente artículo no se enfoca en las dinámicas globales de comercio o las circulaciones de comerciantes individuales. Sino que se concentra en el aspecto más situado de prácticas relacionadas a establecerse, ya sea de manera temporal o más permanente, en lugares de comercio que también tienen un significado social para estas personas migrantes. En São Paulo tales procesos se pueden observar en barrios enmarcados por el comercio y la industria textil como Brás (Amrith, 2018) y en centros comerciales como la Galeria Presidente, en el centro antiguo de la ciudad. La Galeria Presidente no se inserta solamente en un ámbito de comercio influenciado por personas migrantes, sino también en un contexto urbano específico. En São Paulo tales galerías, mayoritariamente construidas en los años 1960, han servido desde hace décadas de puntos de encuentro e intercambio para personas que adscriben a identificaciones comunes o comparten interés en ciertas subculturas urbanas, como describe Magnani con referencia a la Galeria Ouro Fino y su relación con los raves, la Galeria do Rock, o la Galeria Presidente, también llamada Galeria do Reggae (Magnani, 2005; 1996).

Desde la perspectiva de mis interlocutoras, la peluquería en la Galeria Presidente donde trabajaban estaba en relación con otro lugar que formaba parte del mismo ámbito social, una iglesia evangélica. Lugares de comercio y de religión (Pardue 2017), en este caso, estaban estrechamente vinculados. La relación entre prácticas religiosas de orientación evangélica y prácticas del haciendo-lugar se analizó en distintos contextos urbanos. Muchas veces se subrayó cierta relación entre práctica religiosa evangélica y narrativas de expansión territorial de la religión, incluso en el caso de iglesias fundadas o frecuentadas por personas migrantes africanas (Garbin, 2013; Çağlar, Glick Schiller, 2018). En su estudio sobre iglesias evangélicas fundadas por personas migrantes congoleñas, Garbin (2013) resalta el doble esfuerzo de estas personas para afirmar la presencia de sus iglesias en los contextos urbanos donde han migrado, así como en las redes religiosas transnacionales. En el caso estudiado aquí, se considera la iglesia como un lugar donde se crean relaciones sociales jerárquicas y estrechamente vinculadas con relaciones de trabajo. Antes de profundizar en la iglesia, a continuación se explicará primero el contexto de la Galeria Presidente donde encontré a Gabrièle, Thérèse y otras migrantes congoleñas.

\section{La peluquería en la Galeria Presidente}

El Centro Comercial Presidente, popularmente más conocido como Galeria Presidente o Galeria do Reggae está ubicado en el centro antiguo de São Paulo. El centro antiguo es un polo de comercio, frecuentado por aquellos/-as que trabajan ahí, por de las clases populares y turistas durante el día y abandonado por los mismos desde que cae la noche (Earle, 2012). 
No hace parte de las áreas conservadas de la ciudad. Muchos edificios están en un estado de decadencia, mientras que las zonas más ricas de la ciudad se concentran en el suroeste de la metrópoli. La Galeria Presidente no es una excepción. Cuando la visité en 2017, el material de construcción me pareció viejo, los vidrios de algunos locales estaban rajados, y muchas veces las escaleras mecánicas que conectan los pisos del centro comercial no funcionaban.

Este centro comercial, ubicado en un área caracterizada por presencias temporales con objetivos de consumo y tránsito, no constituye, por lo tanto, un no-lugar, en el sentido de Augé (2000). La Galeria Presidente es, como se mostrará más adelante, un lugar sumamente social, dotado de sentido por las relaciones que se crean aquí y las prácticas de las personas que hacen del centro comercial su lugar. Y este carácter social no es algo nuevo. Desde los años 1970 la Galeria Presidente ha sido un punto de encuentro para el movimiento político y cultural de la comunidad negra brasileña y productores de la música black. Se convirtió en un lugar donde se intercambiaron aficionados de diferentes estilos de hip-hop, reggae e, incluso, heavy metal en los años 1990 (Pardue, 2017). En 1996, Magnani describió la Galeria Presidente como un punto de referencia importante para jóvenes negros brasileños quienes se encontraban ahí, no únicamente a fin de consumir, sino "para encontrar seus iguais, exercitar-se no uso dos códigos comuns, apreciar os símbolos escolhidos para marcar as diferenças" (Magnani, 1996, p. 40). Hoy en día, la Galeria es un punto de encuentro importante para personas migrantes africanas (Sato, 2017, p. 120; Pardue, 2017). Muchos de sus locales, sobre todo en los pisos superiores, son alquilados por migrantes de distintos países africanos. La Galeria contiene una mezcla de locales que venden música, accesorios, comida o que ofrecen transferencias internacionales de dinero. Particularmente llamativo es el número de peluquerías y de locales que venden todo tipo de extensiones de pelo sintético y natural. Muchas de las personas que dirigen o trabajan en estas peluquerías son mujeres migrantes africanas. Las peluquerías constituyen un espacio particular- pero no exclusivamente femenino que da oportunidades de empleo a mujeres migrantes. En la actualidad, para muchas personas migrantes africanas la Galeria Presidente es ciertamente su lugar de trabajo, pero también mucho más: es un punto de encuentro al que vienen para sentarse a comer o tomar una cerveza, encontrar a amigos/-as, conocidos/-as y parientes, intercambiar noticias y pasar su tiempo libre (Sato, 2017).

En uno de los niveles superiores de la Galeria Presidente estaba la peluquería donde conocí a Gabrièle. Al lado había un bar-restaurante de dos niveles como las otras tiendas. Abajo se tomaban cervezas y gaseosas, arriba uno se podía sentar y comer recetas congoleñas preparadas por las dos jefas 
del restaurante, ambas provenientes de la RDC. La peluquería de la dueña Maman Tina tenía un pequeño baño en el fondo, al igual que un lavabo que servía para lavar el pelo de los/las clientes y una pequeña mesa de recepción. En una de las paredes laterales había grandes espejos y varios asientos. En las otras paredes había estantes cubiertos con pelo sintético y natural en diferentes colores y estilos y con productos para cuidar, lavar, alisar o pintar el cabello.

En esta peluquería trabajó Thérèse, la hermana menor de Gabrièle que había llegado a Brasil en 2014. En Kinshasa, donde Thérèse había vivido antes de llegar a Brasil, ya había trabajado como peluquera. En São Paulo encontró trabajo en la Galeria Presidente gracias a sus conocimientos. Después de algún tiempo, la dueña Maman Tina, con quien Thérèse había establecido una relación de confianza, le designó "gérante" (gerente) de su peluquería.

Maman Tina era la persona responsable de la compra de todos los productos y del pelo sintético y natural que se podía comprar en su local. Se había establecido toda una red de proveedores, y el pelo que éstos le traían venía mayoritariamente de otras partes de Brasil y de China. Maman Tina era también la única persona que vendía el pelo y los productos expuestos en su local. Durante el día, estaba abajo en la entrada de la Galeria Presidente, que se abre directamente a una zona peatonal. Se sentaba en un taburete de plástico y trataba de atraer a posibles clientes, de vender sus productos y el pelo o de proponerles un peinado. Los peinados que hacían las mujeres en la peluquería contenían todo tipo de trenzas y de extensiones.

La segunda mano de Maman Tina era su gerente, Thérèse. Cada mañana era la primera que llegaba a la peluquería. Entre las seis y las seis y media abría el local, limpiaba, ordenaba y preparaba el espacio para la llegada de los/las primeros/-as clientes. Maman Tina y Thérèse eran las únicas que cobraban y guardaban el dinero de los/las clientes, y anotaban los ingresos y el nombre de la peluquera que había hecho el peinado. Cada sábado, las dos mujeres hacían las cuentas y Maman Tina le pagaba a cada una de las peluqueras lo que ella había ganado durante la semana.

Maman Tina no tenía "empleadas". A las señoras que trabajaban en la peluquería -todas de la RDC y muchas con una experiencia migratoria previa en Angola- Maman Tina les dejaba un espacio para trabajar. También les llevaba clientes desde la calle. El precio que pagaron los/las clientes se componía por el precio del pelo sintético o natural y de los productos necesarios de un lado, y por el precio del peinado por el otro. Además de cobrar el precio de los productos y del pelo, Maman Tina cobraba la mitad del precio de cada peinado hecho en su peluquería. La otra mitad recibía la peluquera. Thérèse, como gerente, no ganó más que cualquiera de las otras peluqueras, por lo 
cual ella me dijo una vez en un tono amargo: "Soy gerente dans la bouche [lit. en la boca] pero en el bolsillo- iNo gano nada, nada!"4.

A pesar de que Thérèse mostraba cierta frustración sobre la desproporción entre su trabajo y su sueldo, ella y las otras peluqueras describieron a Maman Tina, quien había llegado a Brasil como estudiante diez años atrás, como una persona bondadosa. Subrayaron sobre todo su experiencia en cuanto a la vida en Brasil y la importancia de sus consejos. Gabrièle solía decir: "Es Maman Tina quien te dice: Hazlo así, hazlo así, no hagas esto". En relación con su rol de indicadora de camino es importante destacar que Maman Tina no era solamente la dueña del local, sino también la esposa del pastor de la iglesia evangélica "Reobote", visitada por muchas de las peluqueras. Ésa fue también la razón por la que las demás la llamaron "Maman". Por un aviso para un seminario organizado por la iglesia colgado en la vidriera de la peluquería, la iglesia era siempre materialmente presente.

En el día a día de las peluqueras, los papeles de Maman Tina como esposa del pastor y dueña de la peluquería estaban entrelazados, como lo describió la peluquera Gloria: había llegado con su marido y sus dos hijos un año y medio atrás, y su marido había buscado trabajo en vano. Rezando en la iglesia, Gloria había conocido a Maman Tina. "Y después, Maman Pasteur me dijo: Ven aquí, haz esto [el trabajo de peluquera]". Gloria tenía que aprender las técnicas de la peluquería primero, porque nunca había trabajado en esta profesión. Pero gracias a este trabajo, ella y toda su familia habían sobrevivido estos últimos meses. Cuando su marido encontró un empleo como barrendero, ella vinculaba este hecho a sus oraciones regulares en la iglesia de Maman Tina: "Es su Dios [de Maman Tina] quien nos ayudó". Así, las relaciones de trabajo en la peluquería estaban también marcadas por las relaciones en la comunidad religiosa de la cual las mujeres hacían parte. Maman Tina era un punto de anclaje en esta configuración social, un hecho que se me hacía aún más claro cuando participé un domingo en el servicio religioso de la iglesia evangélica Reobote.

\section{Un domingo en la parroquia}

El primer domingo de abril de 2017 me encontré a las diez de la mañana sola en frente de la iglesia Reobote en Cidade Antonio Estevão de Carvalho, no muy lejos de la estación de Corithians-Itaquera. Era la última estación de la línea 3, la línea más frecuentada del metro, en el este de la ciudad, a un poco más de una media-hora de trayecto del centro antiguo. La iglesia era una casa bajita como las demás en la calle pendiente. Era abierta hacia la calle, igual como el bar a su lado izquierdo. A esta hora de la mañana, no había

\footnotetext{
4 A menos que se indique lo contrario, todas las citas son traducidas del francés.
} 
casi nadie, excepto dos personas que yo percibí como sumamente distintas. En el escalón en frente del bar estaba sentada una señora, probablemente brasileña, con ropa ancha y descuidada, y al lado de la iglesia desierta estaba esperando un varón joven, vestido de manera elegante, con camisa, pantalón tenue y gafas con lentes espejados. Empecé a conversar con él. Estaba aquí por primera vez, como yo. Me contaba que era hijo de un padre angolano y de una madre de la República del Congo y que había crecido en Angola.

Poco a poco estaban llegando el pastor y los primeros músicos, todos migrantes congoleños o angolanos, y empezaban a tocar y a rezar. El pastor, así como los músicos usaban micrófonos y amplificadores, y sus voces se escucharon claramente en la calle. Siguiendo a los primeros sonidos, la señora se levantó del escalón y tambaleaba hacia la entrada de la iglesia. Parecía como si hubiese consumido algún estupefaciente. Nosotros también entramos y nos sentamos en unas sillas blancas de plástico orientadas hacia el fondo de la sala, donde se veía una pared decorada con telas coloridas, el atril con la biblia y los músicos que tocaban a su izquierda. Poco a poco la iglesia se fue llenando. Llegaron Thérèse y otras de las peluqueras que trabajaban con Maman Tina, y cuando llegó Gabrièle, se sentó al lado mío. Mientras tanto, los músicos y el pastor continuaban imperturbables con sus oraciones y sus cantos. El pastor habló exclusivamente en lingala, pero todo lo que decía fue inmediatamente traducido al portugués por una señora joven.

Al parecer, la señora brasileña y yo éramos las únicas personas presentes de origen no africano. La señora me llamó una vez más la atención cuando llegamos a una parte del servicio religioso dedicado a la adoración de Dios. Mientras que todos/-as estaban cantando y rezando con sus brazos estirados hacia arriba, ella se levantó de su silla y caminó hacia adelante, donde empezó a comentar el vestido colorado de una señora sentada en la primera fila: "Bonito, né!". Por primera y única vez durante este servicio religioso, el pastor interrumpió su predicación y se dirigió a la señora en portugués: "Pode sentar, por favor". Ella se dejó caer en una silla libre en la primera fila, la silla directamente al lado del pasillo central. En este momento, las demás personas en la iglesia empezaban a intercambiar miradas que yo no entendía. ¿Qué pasó?, me pregunté. Un varón que al inicio fue parte de la banda musical y que después se había dedicado a filmar la predicación se aproximó a la señora y le pidió de elegir otro lugar para sentarse. Yo veía que la tomaba el brazo y la conducía, de manera cortés pero decidida, a una silla más cerca de la entrada. Poco tiempo después entendí por qué la señora tenía que cambiar de silla: Llegó Maman Tina, con un vestido colorado, el pelo suelto y voluminoso, un niño en el brazo y dos niños más caminando a su lado. Ella se dirigió directamente hacia la primera fila. La silla al lado del pasillo central estaba reservada exclusivamente para ella. 
Dentro de la sala llena de personas rezando, cantando y bailando, Maman Tina llamó la atención de todos/-as. Llegó casi en último momento, segura de la silla reservada en la primera fila, y se puso directamente a rezar y a bailar con la música. Movía todo su cuerpo, se tiró al suelo, gritaba y cantaba en voz alta y estridente. En un momento sacó unos billetes de su bolsa y le dio a uno de los músicos, de una manera visible para todo el mundo. Cuando se les pidió el diezmo a los miembros de la parroquia, Maman Tina era la primera en la fila rápidamente formada. Bajo la elación de los demás feligreses, entregó un sobre blanco probablemente lleno de dinero. Thérèse se unió también a la fila y dejó un sobre. Mientras tanto, Gabrièle se quedó sentada.

Las observaciones hechas durante el servicio religioso son interesantes desde una perspectiva del haciendo-lugar, así como del desplazamiento. Primero, esta iglesia mayoritariamente atendida por personas migrantes angoleñas y congoleñas parecía mostrar claramente su presencia a su entorno, ya por ser tan audible en la calle. Refiriéndose a una favela en Rio de Janeiro, Oosterbaan (2009) muestra cómo distintos actores, incluso iglesias evangélicas, compiten por su presencia auditiva en este contexto urbano particular. Según Oosterbaan, el hecho de ser audible significa ser un punto de referencia para, por ejemplo, los adherentes de ciertas iglesias, que se distinguen por sus sonidos de otros agentes audibles, como de los organizadores de fiestas funk. A diferencia del caso descrito por este autor, cuando visité a la iglesia Reobote, era el único agente que llenó el área con sonidos, por lo menos en este momento. Sin embargo, por los sonidos electrónicamente reforzados que retumbaron de la puerta abierta de la iglesia, la comunidad religiosa se hacía no solamente visible, pero sobre todo audible, lo que puede ser interpretado como una afirmación de la presencia de las personas migrantes miembros de la parroquia en este contexto urbano.

Las configuraciones sociales y relaciones de poder dentro de la parroquia se materializaron en el espacio, y el comportamiento de las personas durante el servicio religioso posibilitó sacar conclusiones sobre su posición en las jerarquías de la parroquia. El rol particular de Maman Tina nunca me había parecido más claro que durante este servicio religioso. No era solamente una persona bondadosa, una madre cuidadora, y la esposa del pastor. Maman Tina ofrecía dinero a la parroquia, a los músicos y pasión a Dios. Era una persona de respeto y en una posición de poder en su negocio, así como en la parroquia, dos lugares inseparablemente vinculados. Mientras que Maman Tina tenía su lugar bien definido en la parroquia, la señora brasileña parecía más bien desplazada. Su comportamiento fue aparentemente juzgado como inadecuado, y, además, le faltaba el conocimiento de las reglas implícitas del ordenamiento espacial y social de la iglesia. Ella fue, en un sentido físico, 
sacada de la escena, y dejada más atrás, trasladada a la posición que le correspondía en esta configuración espacial y social.

En otras ocasiones, sobre todo en Brás, observé interacciones entre personas migrantes y brasileñas que se encontraban en el mismo barrio y frecuentaban los mismos bares. Mis conocidos/-as congoleños/-as criticaron la falta de conocimiento de estas personas. Decían que les faltaba "educación" porque tenían ideas muy generales, exóticas o falsas sobre el continente africano. El presidente de una asociación de personas migrantes congoleñas en Brás se quejó de que las personas migrantes recién llegadas terminaron, a veces, en las mismas estructuras de acogida como personas en situación de calle, personas que él describió como "locos". Drotbohm (2016) analizó los encuentros de migrantes recién llegados/-as con "moradores da rua" en estas estructuras de acogida en São Paulo, y constató que los migrantes "sentem-se contrariados e confrontados com tipos particulares de vulnerabilidade e exclusão urbana com a qual eles não se identificam" (Drotbohm, 2016). Que migrantes y personas en situación de calle u otras personas desfavorecidas compartan una experiencia de desplazamiento, no significa que siempre surjan nuevas "sociabilidades urbanas", como aquellas descritas por Glick Schiller y Çağlar (2016), y tampoco significa que esas personas no traten de distinguirse las unas de las otras. Retornando a la situación observada en la iglesia, aquí, el caso era muy claro: Eran los migrantes que se habían hecho, y materialmente construido, su propio lugar. Éste estaba abierto, audible y entendible, gracias a la traducción ininterrumpida, para un público brasileño. Sin embargo, tenía también sus jerarquías y reglas que no podían ser violadas, tampoco por desconocimiento. Este lugar parecía, para las personas migrantes, una oportunidad de celebrar y marcar la presencia de su comunidad, pero quizá también de diferenciarse de otras personas desplazadas con las cuales no se identificaban.

Volvamos a la perspectiva de la recién-llegada Gabrièle. Conocía a la parroquia y a varias personas que atendían el servicio religioso y estaba consciente de ciertas reglas. Al mismo tiempo, me pareció que no podía destacarse, como Maman Tina o su hermana Thérèse quienes contribuyeron financieramente a la parroquia. ¿O sería que Gabrièle no quería destacarse? En el siguiente párrafo veremos que Gabrièle estaba todavía en busca de su lugar no solamente en la parroquia, sino también en la peluquería. Una busca que resultó, a veces, conflictiva.

\section{Gabrièle en busca de su lugar}

Como su hermana menor, Gabrièle venía diariamente a la Galeria Presidente y pasaba sus días sentada en algún asiento dentro de la peluquería. En la peluquería siempre se encontraban varias personas. Cuando venían muchos/-as clientes, la peluquería estaba llena de peluqueras, cada una con 
un/-a cliente, y cuando terminaban su propio trabajo se metieron a ayudar a otra, haciendo algunas trenzas o desenredando y repartiendo el pelo que quedaba a aplicar. Cuando no había clientes, algunas peluqueras salían para buscar clientes afuera al lado de Maman Tina, otras iban a su casa o a hacer alguna compra y otras se quedaban sentadas, como Gabrièle, charlaban, dormían una siesta o conversaban por sus teléfonos.

A pesar del poco tiempo que había pasado en São Paulo, Gabrièle ya se había familiarizado con el centro comercial y su entorno. Era uno de los lugares donde pasó más tiempo, junto a su casa y la iglesia. Conocía los lugares para comer, los precios y los horarios. Conocía también a algunas de las personas que visitaban habitualmente el centro comercial. Estaban los que venían siempre en la tarde al bar para tomar una cerveza (pero Gabrièle no habló mucho con ellos porque no le gustaba lo mucho que ellos solían tomar). Había también un señor anciano, igualmente migrante de la RDC, que venía cargado con sacos grandes llenos de Matembele ${ }^{5}$ que trataba de vender a aquellas personas trabajando en el centro comercial. También venían miembros de la familia, amigos/-as y otros/-as conocidos/-as.

La Galeria era un lugar de llegada y de inserción para personas recién llegadas como Gabrièle. Aquí los contactos se creaban rápidamente; era un lugar donde las personas socializaban e intercambiaban informaciones. Era también un lugar donde personas experimentadas, como Maman Tina, ofrecían ciertas oportunidades para un primer empleo en esta ciudad desconocida para muchas personas migrantes, quienes además en el momento de su llegada todavía no tenían un permiso laboral.

Al mismo tiempo, insertarse le resultó difícil a Gabrièle. Para una persona migrante, el llegar, el "choque de lo nuevo", como nos recuerda Jackson en su ensayo del mismo nombre, puede estar ligado a la experiencia de múltiples riesgos y peligros y traducirse en un profundo sentimiento de miedo (Jackson, 2008, p. 69). Gabrièle no tenía los conocimientos necesarios para empezar a trabajar como peluquera. Como Gloria, Gabrièle tenía que adquirir estos conocimientos, un proceso que no había terminado al final de mi corta estancia en São Paulo. Cuando se daba la ocasión, les ayudaba a las otras peluqueras, terminando trenzas, limpiando el lavabo o colaborando de otras formas. Pero trenzaba muy lentamente y la mayor parte del tiempo no tenía mucho que hacer. Para Gabrièle, su inactividad fue una fuente de inquietud: Le preocupaba que no era capaz de ganar su vida y mostró cierta desilusión. En lingala, me decía "Nazá na poto", y me tradujo: "Estoy en el país de los blancos". No discutiré aquí el comentario sin duda problemático de Brasil como "país de los blancos". Gabrièle se refirió, con esta frase, a ciertas

\footnotetext{
5 Hojas de batata que constituyen una legumbre popular en la cocina de África Central y del Este.
} 
expectativas que había tenido antes de su migración. La palabra "poto" (o "mputu" en kikongo) viene de "Portugal", o de la ciudad "Porto", debido a la presencia de portugueses en la región de la desembocadura del río Congo durante siglos 6 . Hoy en día, "poto" se refiere a Europa, al occidente, o a países específicos como Francia (Massoumou, Queffélec, 2007, p. 312). Gabrièle lo aplicó a Brasil. Me explicó que "nazá na poto" se solía decir cuando una persona migró hacia Europa -una migración vinculada a muchas esperanzas e ideas idealizadas del lugar de destino. Gabrièle sabía que solicitar refugio en muchos países significa recibir por lo menos una pequeña suma de dinero para sostenerse. No era el caso en Brasil. "Cuando no trabajas, no comes", me decía más de una vez. No la preocupaba solo su propia situación, sino también la de su hija de 19 y su hijo de 16 años que seguían viviendo con la madre de Gabrièle en la RDC. Su hija quería entrar en la universidad, pero le faltó el dinero, y Gabrièle había prácticamente gastado sus ahorros para poder juntarse a su hermana en Brasil. Desde una perspectiva transnacional, las personas migrantes tratan de establecerse en donde llegan, entre otros porque a través de poder hacerse su vida aquí, también confirman su presencia en un allá, el lugar en donde está, por ejemplo, su familia que espera el apoyo de aquellas que se fueron (Drotbohm, 2018). Para Gabrièle, no solamente su haciendo-lugar en São Paulo estaba en juego, sino también la vida en la RDC. No sorprende entonces que veía a su situación con cierta inquietud.

Más allá de sus preocupaciones financieras, Gabrièle parecía sufrir del aburrimiento y del sentimiento de no ser útil. Observé que prestó su ayuda cada vez que podía. En estas situaciones se mostró que la Galeria, este lugar de inserción laboral y social, era también un lugar competitivo y conflictivo. Una recién llegada como Gabrièle tenía que definir su lugar a través de sus relaciones con las personas presentes y a través de sus conductas. Algunas de sus iniciativas para echar una mano no eran bien vistas por las demás. En mi último día en São Paulo surgió un conflicto entre Gabrièle y la peluquera Victoire, que tuvo como resultado que las dos mujeres dejaron de hablarse. Según Gabrièle había pasado lo siguiente: este día, Maman Tina había llegado furiosa en la peluquería. Desde su posición en la entrada de la Galeria, había intentado llamar a algunas de las peluqueras, pero ninguna había contestado al teléfono. Para Maman Tina era importante poder llamar en cualquier momento a alguien que estuviese arriba, no solamente porque no quería subir todas las escaleras mecánicas tan frecuentemente paradas, sino también porque a veces venía la fiscalía para controlar las actividades en el centro comercial. Desde su posición en la entrada llamaba a las peluqueras cuando se acercaba la fiscalía, para avisarles. Cuando Maman Tina confrontó a las peluqueras con

\footnotetext{
6 Agradezco al lingüista y especialista de lingala Nico Nassenstein de haberme informado sobre este aspecto.
} 
sus reproches, Gabrièle le había dicho que siempre podía llamar a su celular. Eso había fastidiado a Victoire. "¿Pourquoi tu te mets là comme ça?" - "iPor qué te metes ahí?". Obviamente, las dos mujeres interpretaron la situación de manera diferente. Victoire reprochó a Gabrièle de haber intentado congraciarse de manera exagerada con Maman Tina. Gabrièle, por su lado, me dijo que solamente había querido señalar a Maman Tina que su celular, anteriormente roto, estaba funcionando.

En su estudio sobre personas migrantes libanesas en Australia, Hage sostiene que para lo que llama "home-building", una persona migrante necesita, entre otros, "un sentido de posibilidad" (Hage, 1997, traducción de la autora). Es decir, la oportunidad de desarrollar capacidades o de aproximarse a ciertos ideales, que estén vinculadas a la acumulación de capital cultural o económico, a un desarrollo emocional o a un ascenso social. Desde el inicio de su estadía en São Paulo Gabrièle parecía expresar una fuerte necesidad de ver o de crearse algún sentido de posibilidad, aunque sea mínimo. Habiendo visto cómo Gabrièle estaba todo el tiempo casi inmóvil e incapaz de empezar su vida laboral en São Paulo, me parecía entendible que ella hubiera tratado de aprovechar la interacción con Maman Tina para dar algún sentido a su estadía en la peluquería.

Sea como fuere, este conflicto entre Gabrièle y Victoire muestra las complicaciones de la situación de una recién llegada en un contexto competitivo. Mientras que todas subrayaron cómo Maman Tina les ayudó a hacer su vida en São Paulo, cada una tenía que aprender dónde era su lugar y qué tipo de comportamiento era bien visto. Cuando nosotras nos encontramos Gabrièle todavía estaba luchando con el haciendo-lugar en este entorno en el que las relaciones sociales tenían que ser constantemente negociadas.

\section{A modo de conclusión}

A aquellas personas que llegan a una ciudad como São Paulo, se les presenta un conjunto de configuraciones sociales, políticas, económicas y legales que requieren un proceso de aprendizaje. Los ejemplos desarrollados previamente resaltan algunos aspectos importantes del haciendo-lugar practicado por personas migrantes congoleñas. La Galeria Presidente y otros lugares están visiblemente marcados por las personas migrantes que trabajan o se dedican a otras actividades en ellos. Estas personas establecen sus redes sociales, comerciales y religiosas que incluyen a otras personas migrantes y no-migrantes. Transforman determinados espacios en lugares de encuentro donde se intercambian informaciones y mercadería, donde se comparten comida y conocimientos, donde se junta una comunidad de creyentes, donde se crean nuevas relaciones y se fortalecen las ya existentes. 
Mientras tanto, el haciendo-lugar permanece un proceso complicado y delicado, siempre cerca de un (nuevo) desplazamiento. Ciertos conocimientos pueden acelerar o impedir la inserción de una persona. Poder recurrir a experiencias laborales previas puede ser un aspecto clave a la hora de buscar empleo, sobre todo en un marco de posibilidades muchas veces limitado, y también en cuanto a la accesibilidad legal a un trabajo para una persona recién llegada. Puede ser difícil insertarse en redes existentes, caracterizadas por ideas de ayuda mutua entre personas de la misma nacionalidad, la misma familia, la misma comunidad religiosa o simplemente en la misma situación laboral o migratoria, pero también por relaciones desiguales de poder. Para Gabrièle, así como para su hermana Thérèse, su situación en São Paulo estaba caracterizada por cierta fragilidad. Sin embargo, ellas y otras personas negociaban sus posiciones y luchaban para obtener los conocimientos requeridos, crear redes, transformar lugares y apropiarse de ellos.

Según Pardue:

La complejidad de raíces y rutas [roots and routes] en el mundo contemporáneo no significa que el impacto de la presencia de los migrantes sea insustancial. El uso de la ciudad por los migrantes, ya que sea en enclaves de afinidad o en grupos multiculturales de interés, produce nuevos espacios constitutivos de la ciudad. (Pardue, 2018, p. 816, traducción de la autora)

Sea por una estadía corta o larga: las personas migrantes que viven en o atraviesan ciudades como São Paulo dejan sus huellas. Al fin y al cabo, el enfoque en sus prácticas y sus experiencias relacionadas a procesos de desplazamiento y del haciendo-lugar va más allá del pensamiento en términos de "integración", "asimilación" o "exclusión", basadas en la suposición de que exista una diferencia esencial entre la población local y la población migrante. Al contrario, cierta fluctuación entre el estar y sentirse en o fuera de su lugar no se limita a la experiencia migratoria, aunque la migración puede añadirle otras dimensiones (transnacionales) al desplazamiento y al haciendo-lugar. Esta conclusión abre perspectivas para futuras investigaciones porque posibilita examinar, a través de un mismo lente analítico, procesos y experiencias que comparten personas migrantes y no-migrantes (Çağlar, Glick Schiller, 2018; Drotbohm, Lems, 2018). Eso puede contribuir, como sostiene Hui (2016), a una de-excepcionalización de la condición migratoria.

\section{Bibliografía}

AMRITH, Megha. Tentative friendships among low-income migrants in São Paulo's commercial districts. Urban Studies, v. 55, n. 3, p. 522-537, 2018.

AUGÉ, Marc. Los "no-lugares". Espacios del anonimato. Una antropología de la sobremodernidad. Barcelona: Gedisa, 2000. 
BAENINGER, Rosana; GUIMARÃES PERES, Roberta. Refugiados africanos em São Paulo, Brasil: espaços da migração. Revista Internacional em Língua Portuguesa, n. 24, p. 97-110, 2011.

BJARNESEN, Jesper; VIGH, Henrik. Introduction: The Dialectics of Displacement and Emplacement. Conflict and Society, v. 2, p. 9-15, 2016.

ÇAĞLAR, Ayşe; GLICK SCHILLER, Nina. Migrants and City-Making. Dispossession, Displacement, and Urban Regeneration. Durham, London: Duke, 2018.

CARVALHO, Nilton. Caritas São Paulo divulga dados dos atendimentos do Centro de Referência para Refugiados. Caritas Arquidiocesana de São Paulo (CASP). 20.04.2018. Disponible en: <https://caritassprefugio.wixsite.com/casp/singlepost/2018/04/20/Caritas-S\%C3\%A3o-Paulo-divulga-dados-dos-atendimentosdo-Centro-de-Refer\%C3\%AAncia-para-Refugiados?fbclid=IwAR3sfkCeAvyJ ZzBGpoq7zWRyOn2gnljtg1MzBX3bALuwfe9pFYLHzaoOpAs>. Acceso en: 01.03.2019.

DROTBOHM, Heike. Paredes porosas: Proteção fragmentada em face do deslocamento de migrantes no Brasil. Traducción por Silvia Rodrigues Parrinha. MigraMundo. 2016. Disponible en: < https://migramundo.com/paredes-porosasprotecao-fragmentada-em-face-do-deslocamento-de-migrantes-no-brasil/> . Acceso en: 01.03.2019.

DROTBOHM, Heike. When 'roofless' migrants make their place. Contradictive perceptions of belonging in São Paulo's squats. Allegra Lab. 2018. Disponible en: <http://allegralaboratory.net/when-roofless-migrants-make-their-placecontradictive-perceptions-of-belonging-in-sao-paulos-squats-displacement/> . Acceso en: 01.03.2019.

DROTBOHM, Heike; WINTERS, Nanneke. Transnational Lives en Route: African Trajectories of Displacement and Emplacement across Central America. Working Papers of the Department of Anthropology and African Studies of the Johannes Gutenberg University Mainz, n. 175, 2018.

DROTBOHM, Heike; LEMS, Annika. Introduction: Displacement and New Sociabilities. Allegra Lab. 2018. Disponible en: <http://allegralaboratory.net/ introduction-displacement-and-new-sociabilities/>. Acceso em: 01.03.2019.

EARLE, Lucy. Irregular Urbanization as a Catalyst for Radical Social Mobilization: The Case of the Housing Movements of São Paulo. In: RODGERS, Dennis; BEALL, Jo; KANBUR, Ravi (orgs.). Latin American Urban Development into the 21st Century. Towards a Renewed Perspective on the City. London: Palgrave Macmillan, 2012, p. 145-164.

ESCOBAR, Arturo. El lugar de la naturaleza y la naturaleza del lugar: ¿globalización o postdesarrollo? In: LANDER, Edgardo (org.). La colonialidad del saber: eurocentrismo y ciencias sociales. Perspectivas latinoamericanas. Buenos Aires: CLACSO, 2000, p. 113-143.

ESCOBAR, Arturo. Territorios de diferencia: Lugar, movimientos, vida, redes. Popayán: Envión, 2010. 
GAGO, Verónica. La razón neoliberal. Economías barrocas y pragmática popular. Buenos Aires: Tinta Limón, 2014.

GARBIN, David. The Visibility and Invisibility of Migrant Faith in the City: Diaspora Religion and the Politics of Emplacement of Afro-Christian Churches. Journal of Ethnic and Migration Studies, v. 39, n. 5, p. 677-696, 2013.

GLICK SCHILLER, Nina; ÇAC̆LAR, Ayşe. Displacement, emplacement and migrant newcomers: rethinking urban sociabilities within multiscalar power. Identities, v. 23, n. 1, p. 17-34, 2016.

HAGE, Ghassan. At Home in the Entrails of the West: Multiculturalism, Ethnic Food and Migrant Home-Building'. In: GRACE, Helen; HAGE, Ghassan; JOHNSON, Lesley; LANGSWORTH, Julie; SYMONDS, Michael (orgs.). Home/ World: Space, Community and Marginality in Sydney's West. Annandale: Pluto Press, 1997, p. 99-153.

HUI, Allison. The Boundaries of Interdisciplinary Fields: Temporalities Shaping the Past and Future of Dialogue between Migration and Mobilities Research. Mobilities, v. 11, n. 1, p. 66-82, 2016.

JACKSON, Michael. The Shock of the New: On Migrant Imaginaries and Critical Transitions. Ethnos, v. 73, n. 1, p. 57-72, 2008.

LEMS, Annika. Being-Here. Placemaking in a World of Movement. New York, Oxford: Berghahn, 2018.

MAGNANI, José Gilherme Cantor. Quando o campo é a cidade: fazendo antropologia na metrópole. In: MAGNANI, José Guilherme Cantor; TORRES, Lilian de Lucca (orgs.). Na Metrópole - Textos de Antropologia Urbana. São Paulo: EDUSP, 1996, p. 12-53.

MAGNANI, José Gilherme Cantor. Os circuitos dos jovens urbanos. Tempo Social, revista de sociologia da USP, v. 17, n. 2, p. 173-205, 2005.

MASSOUMOU, Omer; QUEFFÉLEC, Ambroise Jean-Marc. Le français en République du Congo sous l'ère pluripartiste (1991-2006). Paris: Agence universitaire de la francophonie, éditions des archives contemporaines, 2007.

MATHEWS, Gordon. Ghetto at the Center of the World. Chungking Mansions, Hong Kong. Chicago, London: The University of Chicago Press, 2011.

MATHEWS, Gordon; RIBEIRO, Gustavo Lins; VEGA, Carlos Alba (orgs.). Globalization from Below: The World's Other Economy. London: Routledge, 2012.

MINISTÉRIO DA JUSTIÇA. Refúgio em números. 3ra edição. 2018. Disponible en: < https://www.acnur.org/portugues/wp-content/uploads/2018/04/refugio-emnumeros_1104.pdf $>$. Acceso en: 01.03.2019.

OOSTERBAAN, Martijn. Sonic Supremacy. Sound, Space and Charisma in a Favela in Rio de Janeiro. Critique of Anthropology, v. 29, n. 1, p. 81-104, 2009.

ORGANIZACIÓN INTERNACIONAL PARA LAS MIGRACIONES (OIM). Perfil Migratório do Brasil 2009. Geneva: Organización Internacional para las Migraciones (OIM), 2010. Disponible en: < https://publications.iom.int/system/ files/pdf/brazil_profile2009.pdf>. Acceso en: 01.03.2019. 
ORGANIZACIÓN INTERNACIONAL PARA LAS MIGRACIONES (OIM). Panorama Migratorio de America del Sur 2012. Buenos Aires: Organización Internacional para las Migraciones (OIM), 2012. Disponible en: < http://www.iom.int/files/ live/sites/iom/files/pbn/docs/panorama_migratorio_de_america_del_sur_2012. pdf>. Acceso en: 01.03.2019.

PARDUE, Derek. Cities as migrant emplacement. Anthropology news. 2017. Disponible en: <http://www.anthropology-news.org/index.php/2017/04/05/ cities-as-migrant-emplacement/> . Acceso en: 01.03.2019.

PARDUE, Derek. Lies that take place. In: BAENINGER, Rosana; BÓGUS, Lúcia Machado; MOREIRA, Júlia Bertino; VEDOVATO, Luís Renato; FERNANDES, Duval Magalhães; DE SOUZA, Marta Rovery; BALTAR, Cláudia Siqueira; PERES, Roberta Guimarães; WALDMAN, Tatiana Chang; MAGALHÃES, Luís Felipe (orgs.). Migrações Sul-Sul. Campinas: Universidade Estadual de Campinas, 2018, p. 816-822.

PATARRA, Neide Lopes; FERNANDES, Duval. Brasil: pais de imigração? Revista Internacional em Língua Portuguesa, n. 24, p. 65-96, 2011.

PELICAN, Michaela. Urban Lifeworlds of Cameroonian Migrants in Dubai. Urban Anthropology, v. 43, n. 1/2/3, p. 255-309, 2014.

REDIN, Giuliana; BITTENCOURT MINCHOLA, Luís Augusto. Imigrantes senegaleses no Brasil. Tratamento jurídico e desafios para a garantia de direitos. In: MERLOTTI HERÉDIA, Vania Beatriz (org.). Migrações internacionais. O caso dos senegaleses no sul do Brasil. Caxias do Sul - RS: Quatrilho Editora, 2015, p. 201-217.

REIFFEN, Franziska. Bench acquaintances: finding one's place in a Buenos Aires shopping centre. Allegra lab. 2018. Disponible en: < http://allegralaboratory.net/ bench-acquaintances-finding-ones-place-in-a-buenos-aires-shopping-centredisplacement/>. Acceso en: 01.03.2019.

ROSSA, Lya Amanda; MENEZES, Marilda A. Entre migrações e refúgio: migrações sul-sul no Brasil e as novas tipologias migratórias. In: BAENINGER, Rosana; BÓGUS, Lúcia Machado; MOREIRA, Júlia Bertino; VEDOVATO, Luís Renato; FERNANDES, Duval Magalhães; DE SOUZA, Marta Rovery; BALTAR, Cláudia Siqueira; PERES, Roberta Guimarães; WALDMAN, Tatiana Chang; MAGALHÃES, Luís Felipe (orgs.). Migrações Sul-Sul. Campinas: Universidade Estadual de Campinas, 2018, p. 383-401.

RÖSCHENTHALER, Ute; JEDLOWSKI, Alessandro. Introduction: landscapes of opportunity, mobility and entrepreneurial perspectives. In: RÖSCHENTHALER, Ute; JEDLOWSKI, Alessandro (orgs.). Mobility between Africa, Asia and Latin America. Economic Networks and Cultural Interaction. London: Zed, 2017, p. 1-27.

SATO, Miki Takao: Percursos etnográficos em narrativas com mulheres africanas em São Paulo. Atividades como possibilidades econômicas. Revista Africa(s), v. 4, n. 8, p. 115-140, 2017. 
SILVA, Filipe Rezende; LIMA, Cassio Francisco; FERNANDES, Duval Magalhães. $\mathrm{O}$ caso de imigrantes haitianos, congoleses, senegaleses e ganeses e a relação com o mundo do trabalho no Brasil. In: BAENINGER, Rosana; BÓGUS, Lúcia Machado; MOREIRA, Júlia Bertino; VEDOVATO, Luís Renato; FERNANDES, Duval Magalhães; DE SOUZA, Marta Rovery; BALTAR, Cláudia Siqueira; PERES, Roberta Guimarães; WALDMAN, Tatiana Chang; MAGALHÃES, Luís Felipe (orgs.). Migrações Sul-Sul. Campinas: Universidade Estadual de Campinas, 2018, p. 446-462.

SIU, Helen F. Grounding displacement: Uncivil urban spaces in postreform South China. American Ethnologist, v. 34, n. 2, p. 329-350, 2007.

VIEIRA, Dianne Rafael. Do Congo para o Brasil - entre a formação de redes e a busca por trabalho - o caso dos refugiados e solicitantes de refúgio acolhidos no Rio de Janeiro. Cadernos OBMigra, v. 1, n. 3, p. 146-177, 2015. 